\title{
Fondation, fondements et perspective
}

Thérèse Goyet et Marie-Thérèse Sart

\section{OpenEdition \\ Journals}

Édition électronique

URL : http://journals.openedition.org/ccibp/387

DOI : $10.4000 /$ ccibp.387

ISSN : 2493-7460

\section{Éditeur}

Centre international Blaise Pascal

\section{Édition imprimée}

Date de publication : 16 juin 1979

Pagination : 1-3

ISSN : 0249-6674

\section{Référence électronique}

Thérèse Goyet et Marie-Thérèse Sart, «Fondation, fondements et perspective », Courrier du Centre international Blaise Pascal [En ligne], 1 | 1979, mis en ligne le 23 novembre 2015, consulté le 01 mai 2019. URL : http://journals.openedition.org/ccibp/387 ; DOI : 10.4000/ccibp.387

Ce document a été généré automatiquement le 1 mai 2019.

Centre international Blaise Pascal 


\title{
Fondation, fondements et perspective
}

\author{
Thérèse Goyet et Marie-Thérèse Sart
}

Chers amis de Pascal,

J'écris, donc je suis. Ce courrier vous apporte en bien peu d'espace une histoire, une analyse de l'être présent, un appel en faveur de l'être à venir. Et le tout constitue un acte de l'espérance.

L'histoire ne peut-être bien longue puisque la première idée de notre Centre International Blaise Pascal se forma - sans l'appellation - lors du colloque clermontois Méthodes chez Pascal dans l'enthousiasme du débat de clôture, le 15 juin 1976. Les participants heureux saisissaient quelque chose : c'est que leur rencontre avait en ellemême signification et valeur de méthode. D'une manière qu'on ne pouvait encore ni prévoir ni définir, il fallait continuer cette rencontre féconde dont l'enracinement leur était devenu sensible. On aurait pu parler d'un " génie du lieu ». Sans dire son nom, mais sous des formes agréables, n'était-il pas apparu dans les vitrines de l'exposition « Blaise Pascal, son entourage et son œuvre ", qui s'est tenue, du 10 mai au 16 juin 1976, à la Bibliothèque Municipale et Interuniversitaire, entre les belles boiseries, récemment restaurées, de l'évêque Massillon? Alors on avait constaté que «l'entourage et l'œuvre » de Blaise Pascal pouvaient être entièrement présentés par le moyen des documents conservés dans la région d'Auvergne, (à l'exception, naturellement, des " carrosses à cinq sols » faits pour Paris). L'œuvre de ses mains, n'était-elle pas présente en corps, si, l'on peut dire, avec cette machine arithmétique, petite, et très soignée, que nous avaient généreusement apportée des arrière-cousins domiciliés en Auvergne ? Cette machine et un portrait sont venus par tradition familiale et leur filière a pu être reconstituée. (Voir Méthodes chez Pascal, Paris, P.U.F., 1979, planches I, XXV, XXVI, et les commentaires).

Autour de cette exposition révélatrice, la publicité fut bien modeste, et, faute de crédits et de temps, le catalogue n'a pas été publié. Cependant sa valeur démonstrative a été retenue à la fois par les universitaires responsables du Colloque et par les conservateurs de la Bibliothèque. Les uns et les autres ont pensé qu'une vocation "pascalisante » pouvait bien être inhérente à la structure d'une telle bibliothèque. Celle-ci résulte d'une 
convention signée en 1902 par la Ville et par l'Université, qui a mis en commun leurs fonds, leurs personnels, leurs activités. Ce mariage a l'avantage de réunir des richesses et des forces vives qui ailleurs opèrent séparément. La Municipale apportait son très riche fonds ancien venu de la cité épiscopale, de la bibliothèque du Chapitre, de celle de l'évêque Massillon, et surtout des couvents dont la Révolution a confisqué les bibliothèques, et singulièrement des Pères de l'Oratoire qui avaient hérité eux-mêmes de la bibliothèque de Marguerite Périer, dernière survivante des enfants de Gilberte et Florin Périer. L'Universitaire apportait son potentiel de recherche: les instruments de travail fréquemment mis à jour, et les universitaires eux-mêmes en périodique renouvellement. En 1942, un passionné de Pascal, Albert Ojardias (1870-1943) léguait à cette double Bibliothèque ses collections qui nous enrichissaient de pièces rarissimes comme les publications séparées des Provinciales et les factums des curés gallicans. D'ailleurs les conservateurs successifs ont toujours consacré à la bibliographie pascalienne une attention particulière, tissant pour notre CIBP à venir un substrat préhistorique, ce qui porte dans la durée historique une force de continuité.

5 L'appellation "Centre International Blaise Pascal » a été trouvée juste un an après le Colloque, le 15 juin 1977, lors d'un retour sur les lieux de M. Jean Mesnard. Dès ce moment le Président de l'Université Clermont II, M. Pierre Cabanes, et le Sénateur-Maire de Clermont-Ferrand, M. Roger Quilliot, ont fait connaître leur intérêt pour cette réalisation. Deux grands pascalisants, respectivement des États-Unis et du Japon, M. Hugh Davidson et M. Yoichi Maeda, l'assurent de leur patronage. Et l'équipe d'organisation du Colloque de 1976, composée de deux Parisiens, M. Jean Mesnard et M. Philippe Sellier, et de deux Clermontois, $\mathrm{M}^{\mathrm{me}}$ Thérèse Goyet, et $\mathrm{M}$. Dominique Descotes, se tient - cela va sans dire - à son service.

Le temps passe vite et les choses vont lentement. Quant aux réalisations voici où nous en sommes. À l'intérieur de la Bibliothèque $\mathrm{M}^{\mathrm{me}}$ Goyet a commencé le dépouillement $\mathrm{du}$ Catalogue systématique de la Bibliothèque Municipale, établi par Éd. Vimont et publié de 1878 à 1891, la date paraissant excellente pour permettre une vue d'ensemble historique sur l'époque de Pascal et sur l'histoire (religieuse, mentale, politique) de la province sous l'Ancien Régime. À travers cette masse on a recherché les livres dont on peut penser que Pascal les avait lus. Le legs Ojardias a été également prospecté. Enfin, à partir des relevés de l'exposition de 1976 (livres propres à la Bibliothèque et emprunts faits dans la région), $\mathrm{M}^{\mathrm{lle}}$ Dominique Seguin, bibliothécaire, et $\mathrm{M}^{\mathrm{me}}$ Goyet, ont fait l'inspection de tous les exemplaires anciens des textes de Pascal (jusqu'au milieu du XIX ${ }^{\mathrm{e}}$ siècle), en notant tous leurs signes d'individualité. Cette recension est prête à paraître, jointe à une étude de $\mathrm{M}$. Jean Mesnard sur les ressources des Archives départementales du Puy-de-Dôme. L'ensemble forme une "physionomie régionale de Pascal " qui devait être la première composante d'une somme raisonnée de nos possibilités d'accueil pour la recherche. Ce sont des limites budgétaires qui nous contraignent à nous ramener à une lettre d'information.

Comment se dessine donc notre avenir?

8 Dans sa réunion de juillet 1978, le Conseil Régional d'Auvergne a accordé à la Bibliothèque un crédit de dix mille francs « pour la réalisation du Centre International Blaise Pascal ». Le présent Courrier vise donc à recueillir auprès des spécialistes de Pascal les indications les meilleures pour l'établissement d'un programme d'entrée en ordinateur. Nous pourrons alors intégrer les documents déjà repérés et ceux qui nous viendront, de telle 
façon, que les «produits » correspondent le mieux possible à vos besoins. Le catalogue bibliographique serait ainsi aisément tenu à jour.

Les champs privilégiés seraient Pascal en Auvergne et l'histoire de ses amis, tel le jurisconsulte Jean Domat, dont on nous signale des traces inédites. Seulement ces services, en droit illimités comme la capacité d'un ordinateur, supposent qu'en fait on accroisse régulièrement l'information automatisée, c'est-à-dire que le budget de fonctionnement soit régulièrement renouvelé.

10 Pour laisser la plus grande part de nos crédits au lancement de cette première opération, nous avons pris la décision de limiter autant que possible le coût de la présente enquête. Comme de plus il nous faut prévoir des frais de dépouillement, vous comprendrez, chers correspondants, que nous vous demandions de bien vouloir couler vos réponses dans la contrainte de «formes fixes» commodes à classer. Nous vous prions notamment de ne rien écrire au verso des pièces « standard » dont nous vous fournissons.

11 Mais un budget à court terme ne doit pas être cause d'une pensée à court terme. Au contraire, avec des moyens pauvres il faut que la prévoyance de l'imagination augmente l'efficacité. Des démarches qui ne seraient pas pleines de sens, nous ne pouvons pas nous les permettre.

12 Ainsi, de notre institution naissante nous commençons par mettre en route la fonction conservatoire. Mais pas seulement pour nos biens propres. Nous irons hors de la Bibliothèque afin d'intégrer à notre fichier (illimité puisqu'informatique) les pièces originales concernant Pascal, appartenant aux collections publiques et privées, tout particulièrement celles qui se trouvent dans la région d'Auvergne. Nous classerons les diverses productions que vous nous signalerez au moyen des fiches ci-jointes, et nous incorporerons celles que vous nous adresserez.

13 Mais la patrie de Pascal ne prétend pas à contenir son Pascal. Ce qu'elle veut, c'est assurer un lieu de rencontre naturel, un point central pour les pascalisants, qui soit un organe de relation internationale. Il faut, par exemple, que ce Courrier devienne un véritable moyen de correspondance.

14 Ces deux conditions dépendent d'une troisième qui est partant la première. Pour avoir des qualités il faut être vivant! Les meilleurs documents requièrent la vitalité des hommes qui les interrogent pour devenir significatifs. Notre Centre sera dynamique dans la mesure où ses membres l'activeront de leurs suggestions, l'enrichissant de leurs productions personnelles et stimulantes des uns aux autres l'esprit de recherche, collectif ou individuel, toujours en alerte selon le progrès de la pensée et des sciences.

Nous ne vous avons pas dissimulé la faiblesse de nos moyens logistiques. Pour rendre service à la recherche pascalienne, il nous faut absolument arriver à obtenir, pour le CIBP dans la symbiose avec la Bibliothèque, un local propre, et surtout, très prochainement, un documentaliste attaché à son fonctionnement. L'intérêt que vous manifesterez à notre entreprise sera un des meilleurs arguments pour arracher la décision des pouvoirs publics. Disons-nous que l'excuse sur le malheur des temps n'excuse personne. Toujours il a fallu forcer les destinées pour réaliser quelque chose. Répondez-nous rapidement, complètement, et engagez votre imagination. Vous constaterez que de notre côté nous aurons fait le second pas quand vous recevrez le Courrier $n^{\circ}$ 2. (Sera-ce en 1980 ?)

De la société CIBP nous voilà les actionnaires. Nous sommes tous des fondateurs. 
INDEX

Mots-clés : Pascal, CIBP

AUTEURS

THÉRÈSE GOYET

Professeur à l'Université II de Clermont-Ferrand

MARIE-THÉRÈSE SART

Conservateur en Chef de la Bibliothèque Municipale et Interuniversitaire de Clermont-Ferrand 